\title{
BMJ Open A randomised, open-label trial to assess the optimal treatment strategy in early diffuse cutaneous systemic sclerosis: the UPSIDE study protocol
}

Julia Spierings (D) , ${ }^{1}$ Anna van Rhenen, ${ }^{2}$ Paco MW Welsing, ${ }^{1}$ Anne CA Marijnissen, ${ }^{1}$ Ellen De Langhe, ${ }^{3}$ Nicoletta Del Papa, ${ }^{4}$ Daan Dierickx, ${ }^{5}$ Karina R Gheorghe, ${ }^{6}$ Joerg Henes, ${ }^{7}$ Roger Hesselstrand, ${ }^{8}$ Tessa Kerre, ${ }^{9}$ Per Ljungman, ${ }^{10}$ Arjan A van de Loosdrecht, ${ }^{11}$ Erik WAF Marijt, ${ }^{12}$ Miro Mayer, ${ }^{13}$ Marc Schmalzing, ${ }^{14}$ Roland Schroers, ${ }^{15}$ Vanessa Smith, ${ }^{16,17}$ Reinhard E Voll, ${ }^{18}$ Madelon C Vonk, ${ }^{19}$ Alexandre E Voskuyl, ${ }^{20}$ Jeska K de Vries-Bouwstra, ${ }^{21}$ Ulrich A Walker, ${ }^{22}$ Dirk M Wuttge, ${ }^{8}$ Jacob M van Laar ${ }^{1}$

To cite: Spierings J, van Rhenen A, Welsing PMW, et al. A randomised, openlabel trial to assess the optimal treatment strategy in early diffuse cutaneous systemic sclerosis: the UPSIDE study protocol. BMJ Open 2021;11:e044483. doi:10.1136/ bmjopen-2020-044483

- Prepublication history and additional material for this paper is available online. To view these files, please visit the journal online (http://dx.doi.org/10. 1136/bmjopen-2020-044483).

Received 08 September 2020 Revised 15 February 2021 Accepted 18 February 2021
Check for updates

(C) Author(s) (or their employer(s)) 2021. Re-use permitted under CC BY-NC. No commercial re-use. See rights and permissions. Published by BMJ.

For numbered affiliations see end of article.

Correspondence to Julia Spierings;

J.Spierings@umcutrecht.nl

\section{ABSTRACT}

Introduction Systemic sclerosis (SSc) is a chronic, autoimmune connective tissue disease associated with high morbidity and mortality, especially in diffuse cutaneous SSc (dcSSc). Currently, there are several treatments available in early dcSSc that aim to change the disease course, including immunosuppressive agents and autologous haematopoietic stem cell transplantation (HSCT). HSCT has been adopted in international guidelines and is offered in current clinical care. However, optimal timing and patient selection for HSCT are still unclear. In particular, it is unclear whether HSCT should be positioned as upfront therapy or rescue treatment for patients refractory to immunosuppressive therapy. We hypothesise that upfront HSCT is superior and results in lower toxicity and lower long-term medical costs. Therefore, we propose this randomised trial aiming to determine the optimal treatment strategy for early dcSSc by comparing two strategies used in standard care: (1) upfront autologous HSCT versus (2) immunosuppressive therapy (intravenous cyclophosphamide pulse therapy followed by mycophenolate mofetil) with rescue HSCT in case of treatment failure.

Methods and analysis The UPSIDE (UPfront autologous hematopoietic $\underline{\text { Stem }}$ cell transplantation vs Immunosuppressive medication in early DiffusE cutaneous systemic sclerosis) study is a multicentre, randomised, open-label, controlled trial. In total, 120 patients with early dcSSc will be randomised. The primary outcome is eventfree survival at 2 years after randomisation. Secondary outcomes include serious adverse events, functional status and health-related quality of life. We will also evaluate changes in nailfold capillaroscopy pattern, pulmonary function, cardiac MR and high-resolution CT of the chest. Follow-up visits will be scheduled 3-monthly for 2 years and annually in the following 3 years.

Ethics and dissemination The study was approved by the Dutch Central Committee on Research Concerning Human Subjects (NL72607.041.20). The results will be disseminated through patient associations and conventional scientific channels.

\section{Strengths and limitations of this study}

- This study is a multicentre, randomised, controlled, open-label trial aiming to determine whether upfront autologous stem cell transplantation (HSCT) is superior to standard immunosuppressive treatment (with rescue HSCT for those who progress) in early diffuse cutaneous systemic sclerosis (dcSSc).

- Event-free survival is the primary outcome measure and is defined as the time in days from randomisation until death due to any cause or the development of persistent major organ failure (heart, lung or kidney).

- This clinical trial has the potential to change clinical practice in early dcSSc worldwide

Trial registration numbers NCT04464434; NL 8720.

\section{INTRODUCTION}

Systemic sclerosis (SSc) is a debilitating and incurable autoimmune connective tissue disease. Clinical features include vasculopathy, fibrosis and inflammation of skin and internal organs. ${ }^{1}$ Presentation and disease course are very heterogeneous. In the diffuse cutaneous subset of SSc (dcSSc), there is generalised skin thickening and often multiorgan involvement. ${ }^{2-4}$ Due to its progressive character, the median 5-year and 12-year mortality for dcSSc is $\sim 25 \%$ and $70 \%$, respectively. ${ }^{56}$ To prevent progression and death in patients with SSc, it is key to identify individuals at risk at an early stage of the disease and initiate immunomodulating treatment. Methotrexate, mycophenolate mofetil (MMF) and cyclophosphamide 
(CYC) are commonly used in dcSSc, dependent on the organ system involved.

In three randomised controlled trials in dcSSc, treatment with autologous haematopoietic stem cell transplantation (HSCT) improved survival, quality of life and skin fibrosis and prevented disease progression when compared with CYC pulse therapy. ${ }^{7-9}$ In addition, three systematic reviews were published on the efficacy and safety of HSCT in SSc. ${ }^{10-12}$ In all reviews, it was concluded that HSCT provided a survival benefit, improved skin involvement and stabilised pulmonary function compared with intravenous CYC for 12 months. Heterogeneity between studies, however, prevented meta-analysis, yet a trend towards better outcome was observed in patients with a shorter disease duration before HSCT. HSCT has since been implemented in (inter) national treatment guidelines for SSc, made its way into regular clinical care and is reimbursed in several European countries. ${ }^{13}{ }^{14}$ However, recommendations regarding the optimal use and especially the preferred timing of HSCT and the efficacy of other therapies in the course of dcSSc are lacking. Particularly, it is unclear whether HSCT should be positioned as upfront or as rescue treatment for patients not responding to immunosuppressive therapy such as MMF or intravenous pulse CYC.

HSCT as upfront treatment might result in better outcomes because the disease process is effectively targeted early in the disease at a time when there is less irreversible organ damage. On the other hand, HSCT is a treatment associated with a higher risk of adverse events compared with other treatments, as it is associated with a treatmentrelated mortality of approximately $10 \% .{ }^{815}$ Even so, there might be fewer transplant-related adverse events due to the better health status of patients and limited immunosuppressive premedication if HSCT is commenced early, compared with the patients who need rescue HSCT after months or even years of other immunocompromising agents. So to determine the optimal treatment strategy in early dcSSc, further investigation is needed.

This article describes the protocol of the UPSIDE (UPfront autologous hematopoietic Stem cell transplantation vs Immunosuppressive medication in early DiffusE cutaneous systemic sclerosis) study. The UPSIDE study is a multicentre, randomised, open-label, controlled trial that aims to compare two treatment strategies used in standard care of adult patients with early dcSSc: upfront autologous HSCT versus intravenous CYC pulse therapy, followed by oral MMF and rescue HSCT in case of treatment failure. Efficacy, safety, survival and cost-effectiveness will be evaluated.

\section{Aims and objectives}

The UPSIDE study aims to investigate the optimal timing of HSCT in early dcSSc by comparing two treatment strategies: the effect of HSCT as upfront therapy compared with that of immunosuppressive medication and HSCT in case of failure, with respect to (eventfree) survival and prevention of major organ failure, safety and the impact on skin thickening, visceral involvement, functional status and quality of life.

\begin{tabular}{ll}
\hline Table 1 Participating centres \\
\hline Country & Centres and affiliated networks \\
\hline The Netherlands & $\begin{array}{l}\text { Amsterdam UMC } \\
\text { Leiden University Medical Centre } \\
\text { Radboudumc Nijmegen } \\
\text { University Medical Centre Utrecht } \\
\text { (coordinating centre) }\end{array}$ \\
& National scleroderma network: Arthritis \\
& Research and Collaboration Hub (ARCH) \\
& University Hospital Ghent \\
Belgium & University Hospital Leuven \\
& National scleroderma network: Belgian \\
& Scleroderma Cohort \\
Germany & Ruhr University Bochum \\
& University Hospital Freiburg \\
& Universitats Klinikum Tuebingen \\
& Universitats Klinikum Wurzburg \\
Kweden & Karolinska University Hospital \\
& Stockholm \\
& Skåne University Hospital Lund \\
Switzerland & University Hospital Basel \\
Italy & ASST G.Pini-CTO, Milano \\
Croatia & University Hospital Zagreb \\
&
\end{tabular}

Secondary goals are to evaluate (in both treatment arms) whether disease activity correlates with immunological parameters, including immunopathology of skin, immune reconstitution and autoantibodies. Cost-effectiveness of both therapeutic options and factors associated with response to treatment will also be examined.

\section{METHODS AND ANALYSIS}

This protocol has been developed in accordance with the Standard Protocol Items: Recommendations for Interventional Trials 2013 statement (https://www.spirit-statement.org/).

\section{Study design and setting}

The UPSIDE study is a randomised, controlled, multicentre, open-label trial. SSc is a rare condition, and stem cell mobilisation after intravenous CYC administration and HSCT are treatments only performed in experienced tertiary treatment centres. Therefore, national and international collaboration is of key importance to include the necessary number of patients. Participants will be recruited from 15 participating centres from the Netherlands, Belgium, Germany, Italy, Sweden, Switzerland and Croatia (table 1). Multidisciplinary expert teams in the field of SSc and HSCT are involved in each centre.

\section{Study population and eligibility criteria}

We will include 120 patients with early dcSSc who fulfil the inclusion criteria (table 2). These criteria are designed to select patients in an early stage of the disease, but at high risk of disease progression and subsequent death. Baseline 
Table 2 Inclusion and exclusion criteria

\begin{tabular}{|c|c|}
\hline Inclusion criteria & Exclusion criteria \\
\hline Age between 18 and 65 years & Pregnancy or unwillingness to use adequate contraception during study \\
\hline Fulfilling the 2013 ACR-EULAR classification criteria for dcSSc & Poor compliance of the patient as assessed by the referring physician \\
\hline
\end{tabular}

Written informed consent
Previous treatments with immunosuppressants $>6$ months, including mycophenolate mofetil, methotrexate, azathioprine, rituximab, tocilizumab and glucocorticoids

Previous treatments with TLI, TBI or alkylating agents including cyclophosphamide

Significant exposure to bleomycin, tainted rapeseed oil, vinyl chloride, trichlorethylene or silica

Eosinophilic myalgia syndrome, eosinophilic fasciitis, morphea

ACR-EULAR, American College of Rheumatology/European League Against Rheumatism; AV, atrioventricular; BP, blood pressure; dcSSC, diffuse cutaneous systemic sclerosis; DLco, diffusing capacity for carbon monoxide; ECOG, Eastern Cooperative Oncology Group; FVC, forced vital capacity; HR-CT, high-resolution CT; HTLV, human T-lymphotropic virus; LVEF, left ventricular ejection fraction; mPAP, mean pulmonary artery pressure; mRSS, modified Rodnan skin score; TBI, total body irradiation; TLC, total lung capacity; TLI, total lymphoid irradiation.

assessment prior to randomisation includes complete blood count, liver function, kidney function, urine spot test (protein/creatinine ratio), viral serology tests, 12-lead ECG, cardiac ultrasound, cardiac MR, right heart catheterisation, high-resolution CT of the chest and pulmonary function tests. Patient recruitment started in September 2020. We anticipate the accrual time will be 3 years. Patients will be followed for 5 years.

\section{Randomisation}

Eligible patients who provide informed consent will be randomised 1:1 (variable block randomisation) using the validated algorithm within CASTOR, the Electronic Data Capture program used for the study. Blocks of two, four or six patients will be randomly and blindly assigned, stratified by the participating centre. The two treatments are as follows: strategy arm A-upfront high-dose nonmyeloablative autologous HSCT or strategy arm Bintravenous pulse therapy with CYC followed by at least 12 months of oral MMF daily and thereafter HSCT as rescue option. The treatment allocation will be coordinated by the principal investigator of the study site. The investigators and participants are not blinded to treatment allocation.

\section{Interventions}

Arm A: Upfront autologous HSCT

Autologous, non-myeloablative HSCT comprises the following consecutive steps: 
A. Mobilisation: Peripheral blood stem cells (PBSCs) will be mobilised using a regimen consisting of infusion of CYC $2 \mathrm{~g} / \mathrm{m}^{2}$ for 1 day. Hyperhydration, alkalinisation of the urine and Mesna will be given to prevent haemorrhagic cystitis. The patients will receive filgrastim (granulocyte colony-stimulating factor) $5 \mu \mathrm{g} / \mathrm{kg} /$ day subcutaneously once or twice a day for 5 days (or more when necessary), according to local practice.

B. Leukapheresis: Start of leukapheresis is required at a CD34+ cell count of $\geq 10-20 / \mu \mathrm{L}$, according to local practice. This is expected to occur on day 5 or day 6 of filgrastim treatment. Leukaphereses will be performed with the goal to obtain at least $6 \times 10^{6} \mathrm{CD} 34+$ cells $/ \mathrm{kg}$ body weight. The primary goal is to obtain a target dose of $6 \times 10^{6} \mathrm{CD} 34+$ cells $/ \mathrm{kg}$, with a minimum of $2 \times 10^{6} \mathrm{CD} 34+$ cells $/ \mathrm{kg}$ after CD34 selection. The apheresis product will be $4-5^{\log } \mathrm{T}$-cell depleted. The CD34+-selected cells will be cryopreserved and stored in liquid nitrogen until reinfusion. In case of mobilisation failure, the patient will be treated with daily subcutaneous injection of filgrastim $20 \mu \mathrm{g} / \mathrm{kg}$.

C. Prior to conditioning: Echocardiography, troponin and N-terminal pro-brain natriuretic peptide (NTproBNP) should be repeated prior to conditioning to evaluate possible subclinical cardiac toxicity caused by CYC administered during mobilisation. Conditioning can be initiated if left ventricular ejection fraction (LVEF) is $>45 \%$ or has not decreased with $>15 \%$ compared with premobilisation, and there are no uncontrolled arrhythmias. $^{16}$

D. Conditioning: Conditioning is to be initiated preferably within 6 weeks after successful harvest. The conditioning regimen consists of CYC $50 \mathrm{mg} / \mathrm{kg} /$ day intravenously for 4 consecutive days (total $200 \mathrm{mg} / \mathrm{kg}$ ) and rabbit antithymocyte globulin (rbATG, Thymoglobulin). The first dose of CYC will be given on day -5 (day $0=$ day of infusion of PBSC). Hyperhydration, alkalinisation of the urine and Mesna will be given to prevent haemorrhagic cystitis. A total dose of $7.5 \mathrm{mg} / \mathrm{kg}$ intravenous rbATG will be administered over 3 days. Intravenous methylprednisolone $2 \mathrm{mg} / \mathrm{kg}$ will be given on the days rbATG will be administered to improve tolerability of the ATG.

E. Peripheral stem cell infusion: The interval between the last dose of CYC and infusion of the graft will be at least 48 hours. On day 0, CD34+ -selected stem cells are thawed and infused according to local standard operating procedures. The number of CD34+ cells to be reinfused should be $\geq 2.0 \times 10^{6} / \mathrm{kg}$, and residual Tcell content is targeted at $\leq 1.0 \times 10^{5} \mathrm{~T}$ cells $/ \mathrm{kg}$, calculated before freezing. ${ }^{17}$ Exceptional release according to local practice is allowed and will be registered in the electronic case report form.

Arm B: CYC followed by MMF and HSCT as rescue option

Immunosuppressive therapy in arm B consists of 12 -monthly intravenous pulses of CYC $750 \mathrm{mg} / \mathrm{m}^{2}(=9 \mathrm{~g} /$ $\mathrm{m}^{2}$ cumulative) followed by at least 12 months of oral
MMF daily (3g as maximum daily dosage) or mycophenolic acid (up to $2.160 \mathrm{~g}$ daily), according to local practice. Hyperhydration, alkalinisation of urine and Mesna are recommended during 12-monthly intravenous pulses of CYC and will be given according to local protocols to prevent haemorrhagic cystitis.

\section{Supportive care}

Supportive care measures, including prophylactic or therapeutic antibiotics, antiviral or antifungal agents, transfusions and antiemetic agents, will be taken according to local standard operating procedures for such patients. In case of HSCT, particular attention will be paid to the risk of Epstein-Barr virus (EBV) and cytomegalovirus (CMV) reactivation. EBV and CMV load will be monitored by PCR, weekly in the first 3 months following the transplantation and then monthly for the next 9 months. In case of reactivation, the patient will be treated according to standard of care guidelines.

Initiation of an ACE-inhibitor prior to HSCT is strongly recommended (ie, enalapril $5 \mathrm{mg}$ once a day) to prevent scleroderma renal crisis based on the clinical experience from the Autologous Stem cell Transplantation International Scleroderma (ASTIS) trial. ${ }^{8}$ Monitoring of blood potassium levels after initiation of ACE-inhibitors is recommended, especially when combined with co-trimoxazole.

\section{Study outline}

An overview of the study outline is shown in figure 1 (study flow diagram). After randomisation, concurrent immunosuppressive therapy will be discontinued. Glucocorticoids may be continued at the lowest possible dose. Either treatment is to be initiated within 6 weeks after randomisation, that is, mobilisation in patients randomised to arm $\mathrm{A}$ and the first pulse of CYC in patients randomised to arm B.

Rescue therapy may be considered in both arms in case of insufficient response or clinically relevant flare, but preferably not within the first 6 months after randomisation. For patients from arm A, methotrexate, MMF or mycophenolic acid, or rituximab can be (re)instituted, according to local preference. Based on earlier studies, the clinical benefits of intravenous pulse CYC may take between 6 months and 12 months. Therefore, it is recommended to then switch patients from arm B to HSCT only in case of rapidly progressive disease, which is arbitrarily defined as $\geq 30 \%$ increase in modified Rodnan skin score (mRSS) or $\geq 20 \%$ relative decline in forced vital capacity (FVC), total lung capacity (TLC), or diffusion capacity for carbon monoxide (DLco) predicted.

\section{Outcomes and follow-up}

The primary end point of the study will be event-free survival. Event-free survival is defined as the time in days from the day of randomisation until the occurrence of death due to any cause or the development of persistent major organ failure (heart, lung, kidney) defined as follows:

- Heart: LVEF <30\% by cardiac MR (or cardiac echo). 




Figure 1 Study flow diagram. ATG, anti-thymocyte globulin; CYC, cyclophosphamide; HSCT, haematopoietic stem cell transplantation; MMF, mycophenolate mofetil.

- Lungs: respiratory failure=resting arterial oxygen tension $<8 \mathrm{kPa}(<60 \mathrm{~mm} \mathrm{Hg})$ and/or resting arterial carbon dioxide tension $>6.7 \mathrm{kPa}(>50 \mathrm{~mm} \mathrm{Hg})$ without oxygen supply or need of oxygen supply.

- Kidney: need for renal replacement therapy (ie, dialysis)

Secondary outcome measures include the following:

1. Progression-free survival, defined as the time in days since the day of randomisation until any of the following relative changes from baseline has been documented: death, $\geq 10 \%$ drop in (F)VC predicted and/or $\geq 15 \%$ drop in DLco predicted, ${ }^{18} \geq 15 \%$ drop in LVEF by echo or cardiac MR, $\geq 15 \%$ drop in body weight, $\geq 30 \%$ drop in creatinine clearance, $\geq 25 \%$ and $>5$-point increase in skin score and $\geq 0.5$ increase in Scleroderma Health Assessment Questionnaire (SHAQ).

2. Treatment-related mortality, defined as any death during the study period following randomisation that cannot be attributed to progression of the disease according to the consensus opinion of the Data and Safety Monitoring Board (DSMB).

3. Overall survival.

4. Treatment toxicity and adverse events, using WHO toxicity parameters ( $\geq$ grade 3 toxicity) and BMT-CTN (Bone Marrow Transplant Clinical Trials Network, for viral infections) during the study period.
5 . The area under the curve (AUC) of the combined response index for systemic sclerosis (CRISS) over time, measuring the "predicted probability of being improved' over 2 years. This AUC is calculated based on four repeated measures $(6,12,18$ and 24 months) with back-translation to the original scale between 0 and 1 .

6 . The change from baseline over time (ie, during followup) of the following parameters: mRSS; pulmonary involvement: DLco and DLco/VA, (F)VC, TLC, residual volume, mean pulmonary artery pressure by cardiac echo (or right heart catheterisation), lung density measurement by thoracic CT and fluorine-18 fluorodeoxyglucose positron emission tomography scan of lung; renal involvement: urine spot test-creatinine/ protein ratio; myocardial involvement: left ventricular function as measured by cardiac MR; body weight $(\mathrm{kg})$; changes in nailfold capillaroscopy; changes in modified Hand Mobility in Scleroderma (functional assessment of hand function); quality of life (EuroQol five dimensions five levels); SHAQ including visual analogue scale for scleroderma-specific symptoms; gastrointestinal symptom scale (University of California, Los Angeles Scleroderma Clinical Trial Consortium Gastrointestinal Tract Instrument 2.0); sexual functioning (International Index of Erectile Function-15 questionnaire (in men) and Sexual Functioning 
Questionnaire-28 (in women)); fatigue score (Functional Assessment of Chronic Illness Therapy questionnaire); self-assessment of skin (Patient selfAssessment of Skin Thickness in Upper Limb); productivity losses due to health issues (customised iProductivity Cost Questionnaire questionnaire); and characteristics of the immune system: autoantibody concentration and avidity targeting host nuclear antigens, primarily focusing on anti-topoisomerase and anti-RNA polymerase III antibodies (ATA and ARA) ; isotype usage; isotype levels; Fc-glycosylation profiles of anti-topoisomerase and skewing of T-cell receptor repertoire and determination of human leukocyte antigen profiles; inflammatory and fibrotic characteristics of the skin; and levels of ATG in relation to changes in lymphocyte subsets and outcomes.

Follow-up appointments will be according to regular care: monthly the first half year, then 3-monthly until 2 years after randomisation, followed by annual appointments for 3 years (table 3 ).

\section{Statistical analyses}

\section{Sample size}

The sample size is determined assuming a median event-free survival of 2 years in the control group and an (approximate) exponential survival curve based on the survival observed in the HSCT arm of the ASTIS trial. ${ }^{7}$ We expect our proposed intervention to result in a considerable improvement (assumed HR of 0.5) and take a total study period of 5 years (3years for recruiting patients at a constant rate), $10 \%$ loss to follow-up after 5 years in both groups and an alpha of 0.05 into account. Based on the above, we will need 60 patients per group to have at least $80 \%$ power to detect a difference as calculated using the SAS power procedure (two-sample survival, log-rank test). Based on the incidence of dcSSc and the collective treatment experience of the 15 trial sites, we anticipate we can enrol the required 120 patients (60 per group) within 3years. ${ }^{19} 20$

\section{Primary outcome}

Data will be analysed on an intention-to-treat (ITR) basis. Data regarding adverse events and serious adverse events will be provided using descriptive statistics and tables. Population characteristics will be provided using descriptive statistics. To compare event-free survival (the primary end point) and other time-to-event outcomes between treatment groups, Kaplan-Meier (KM) curves will be constructed (based on first event) and tested using the log-rank test and Cox regression to take important prognostic covariates (sex, age, smoking status, cardiac function) and centre (stratification factor for randomisation), as determined a priori (before database lock) in SAP, into account. For all time-to-event outcomes, data are censored at the last visit. Based on a visual inspection of the KM curves, also a treatment $\times$ time interaction will be modelled in the Cox regression analysis to allow for nonconstant hazards over time. An ITT analysis (primary) and per- protocol (PP) analysis will be performed. In the primary analysis for patients leaving the study early (early dropout), this last visit will be treated as the censoring date, and a multiple imputation method for sensitivity analyses of time-to-event data accounting for possible informative censoring will also be performed.

\section{Secondary outcomes}

Secondary continuous outcomes (ie, change from baseline in CRISS score) ${ }^{21}$ measured over time will be analysed using mixed modelling approaches, if needed, based on graphical inspection including a treatment $\times$ time interaction, controlling for important prognostic covariates (sex, age, smoking habit) and centre (stratification factor for randomisation). For binary outcomes at a fixed time point, frequencies and proportions will be calculated and differences tested using $\chi^{2}$ or Fisher's exact tests. The effects of covariates will be evaluated using logistic regression. An ITT analysis (primary) and PP analysis will be performed. A cost-effectiveness analyses will be performed from a societal perspective, including direct medical and non-medical and productivity costs. Cost per quality-adjusted life year gained as well as costs per life year and costs per event-free life year gained will be calculated. The economic evaluation will be done at 5 years in line with the duration of the trial, and the evaluation will be performed in line with the Dutch guidelines for economic evaluations. ${ }^{22}$

\section{Interim analysis}

There will be an interim analysis at 12 months after the first inclusion and/or after 60 patients have been included, whatever comes first, and at the DSMB request. Formal statistical methods for evaluating interim efficacy and toxicity results will be used as guidelines rather than absolute rules. An alpha-spending function (O'BrienFleming) will be used. Reasons for DSMB decisions will be recorded.

\section{Safety}

The UPSIDE trial will be overseen by an international DSMB. The DSMB consists of clinicians (including experts on SSc and stem cell transplantation) and a biostatistician. Every 6 months, the DSMB will review the status and conduct of the clinical trial, evaluate all causes of death and adverse events, and make recommendations to the clinical research group concerning the trial's continuation and modification.

Data collection in the study will be monitored by an independent monitor within Julius Centre, UMC Utrecht, the Netherlands. There will be five scheduled visits per centre; the first visit will be the initiation visit, and thereafter once-a-year visits will be performed. The last visit is combined with the close-out visit.

\section{Patient and public involvement}

Our research question originates from clinical practice and a prior study that investigated patient's experiences, which showed that the lack of evidence to support the 
Table 3 Data collection

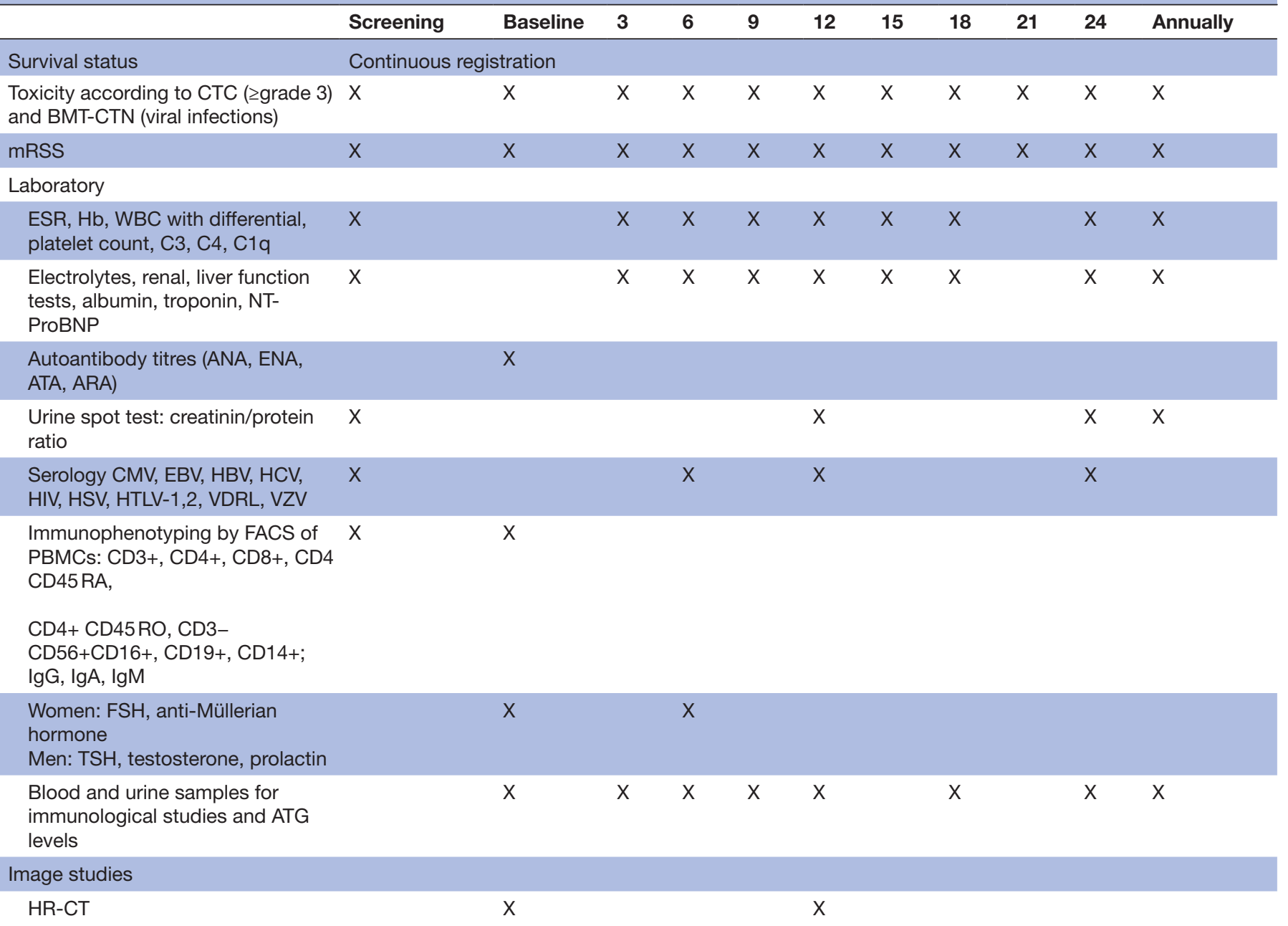

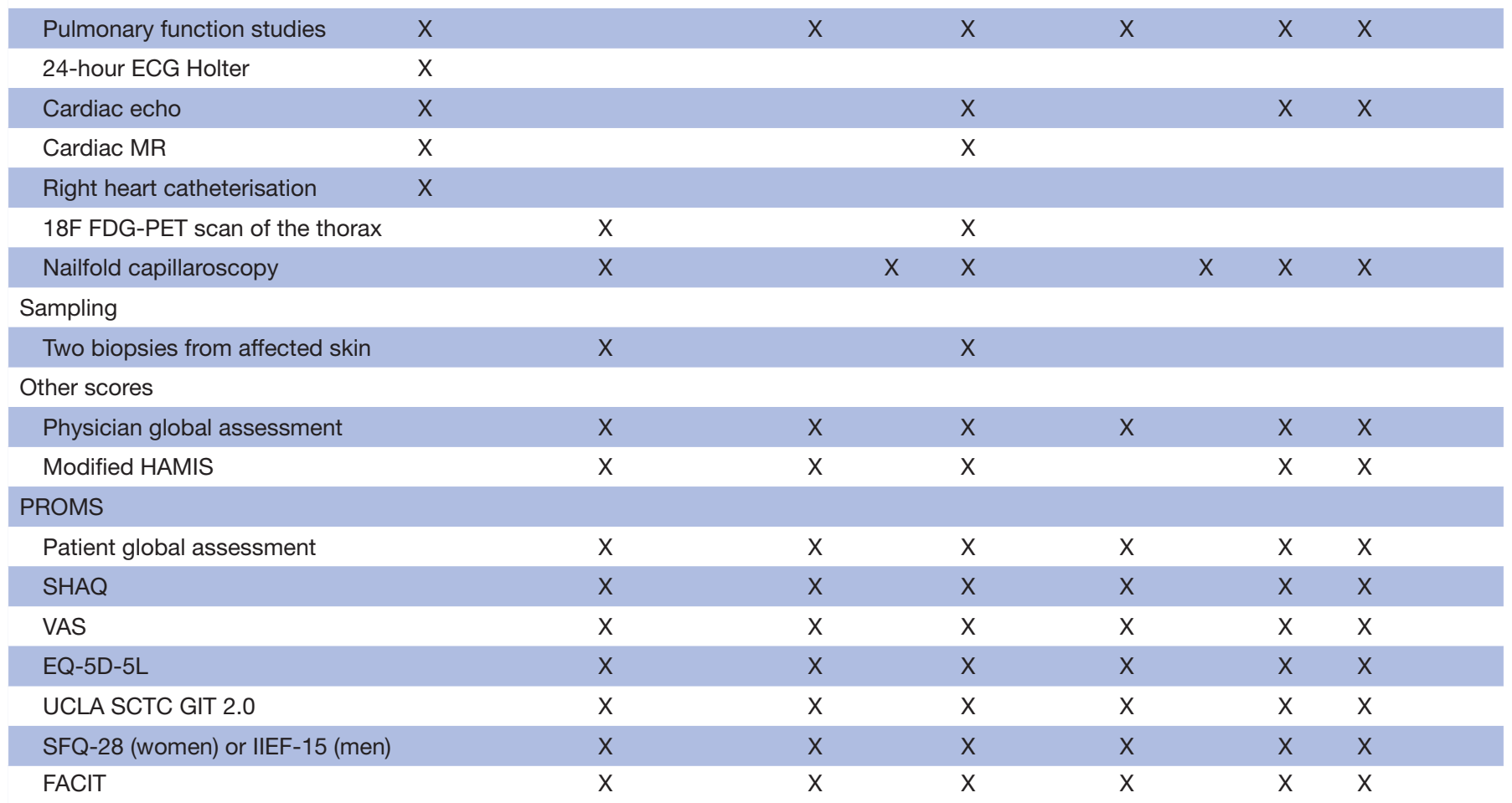


Table 3 Continued

\begin{tabular}{|c|c|c|c|c|c|c|c|c|c|c|c|}
\hline & Screening & Baseline & 3 & 6 & 9 & 12 & 15 & 18 & 21 & 24 & Annually \\
\hline Customised iPCQ & & $x$ & & $x$ & & $x$ & & $x$ & & $x$ & $x$ \\
\hline PASTUL (self-assessment of skin) & & $x$ & $\mathrm{X}$ & $\mathrm{X}$ & $x$ & $\mathrm{X}$ & $\mathrm{X}$ & $\mathrm{X}$ & $\mathrm{X}$ & $\mathrm{X}$ & $x$ \\
\hline
\end{tabular}

ANA, antinuclear antibody; ARA, anti-RNA polymerase III antibodies; ATA

, anti-topoisomerase antibodies; ATG, antithymocyte globulin; BMT-CTN, Bone Marrow Transplant Clinical Trials Network; CMV, cytomegalovirus; CTC, common toxicity criteria; EBV, Epstein-Barr virus; EQ-5D-5L, EuroQol five dimensions five levels; ESR, estimated sedimentation rate; FACIT, Functional Assessment of Chronic Illness Therapy; FDG-PET, fluorodeoxyglucose-positron emission tomography; FSH, follicle stimulating hormone; HAMIS, Hand Mobility in Scleroderma; Hb, haemoglobulin; HBV, hepatitis B virus; HCV, hepatitis C virus; HR-CT, high-resolution CT; HSV, herpes simplex virus; IIEF-15, International Index of Erectile Function; iPCQ, iProductivity Cost Questionnaire; mRSS, modified Rodnan skin score; NTProBNP, N-terminal pro-brain natriuretic peptide; PASTUL, Patient self-Assessment of Skin Thickness in Upper Limb; PBMCs, peripheral blood mononuclear cells; PROM, patient-reported outcome measure; SFQ-28, Sexual Functioning Questionnaire; SHAQ, Scleroderma Health Assessment Questionnaire

; TSH, thyroid stimulating hormone; UCLA SCTC GIT, University of California, Los Angeles Scleroderma Clinical Trial Consortium Gastrointestinal Tract Instrument; VAS, visual analogue scale; VDRL, Venereal Disease Research Laboratory; VZV, varicella zoster virus; WBC, white blood count.

decision-making process in choosing the right treatment strategy is a burden for patients and caretakers. ${ }^{23} \mathrm{~A}$ patient panel (International Panel of Representatives of Patient Organizations and Patient Partners) was involved in the design of this study, particularly in the selection of outcome measures and questionnaires and development of patient information and the consent form. Also, they assessed the burden of the interventions evaluated in this study. The panel will continue to be involved in the 6-monthly evaluation of the study progress and will support dissemination of information about the study among potential participants. After completion of the study, we intend to write a patient summary and publish and disseminate the results using media accessible by patients (including the magazine of patient organisations, social media and the study website). The Dutch patient organisation for systemic sclerosis (NVLE) recognises the importance of the research question and supports this study.

\section{Ethics}

The study will be performed according to the principles of the Declaration of Helsinki (adopted by the 18th World Medical Association (WMA) General Assembly, Helsinki, Finland, June 1964 and amended by the 64th WMA General Assembly, Fortaleza, Brazil, October 2013) and in accordance with the Dutch Medical Research Involving Human Subjects Act (WMO).

Patient information will be handled with care, taking into consideration the required confidentiality as stated by the Law for the Protection of Personal Information, the Dutch Medical Treatment Contracts Act, the EU General Data Protection Regulation and the Dutch Act on Implementation of the General Data Protection Regulation. All data will be stored in a pseudonymised database (CASTOR). A limited number of people have access to the data. They are the principal investigator, coordinating investigator and data manager. Personal data are only processed by the researchers or by those who fall directly under their authority. In addition, the study monitor (Clinical Research Associate), auditors, employees from the Medical Research Ethics Committee and the Health Care Inspectorate of the Ministry of Health, Welfare and Sport have access to the source data. All are subject to the pledge of confidentiality. The data are directly imputed in CASTOR and securely stored. Research data will be kept up to 15 years after ending the research.

\section{Dissemination}

The results will be presented on scientific conferences and through publication of articles in peer-reviewed and patient journals. After publication of the main study results, the study data will be made available on request. The study protocol, statistical analysis plan and informed consent form will be made available as well (see online supplemental material).

\section{Author affiliations}

${ }^{1}$ Department of Rheumatology \& Clinical Immunology, UMC Utrecht, Utrecht, The Netherlands

${ }^{2}$ Department of Haematology, UMC Utrecht, Utrecht, the Netherlands

${ }^{3}$ Department of Rheumatology, KU Leuven Hospital, Leuven, Flanders, Belgium ${ }^{4}$ Department of Rheumatology, ASST Gaetano Pini, Milano, Lombardia, Italy

${ }^{5}$ Department of Haematology, KU Leuven Hospital, Leuven, Flanders, Belgium

${ }^{6}$ Department of Rheumatology, Department of Medicine, Karolinska University Hospital, Karolinska Institutet, Stockholm, Sweden

${ }^{7}$ Department for Internal Medicine (Oncology, Haematology, Rheumatology and Immunology), University Hospital Tubingen, Tubingen, Baden-Württemberg, Germany

${ }^{8}$ Department of Clinical Sciences, Lund, Section for Rheumatology, Lund University and Skåne University Hospital, Lund, Skåne, Sweden

${ }^{9}$ Department of Haematology, University Hospital Ghent, Gent, Oost-Vlaanderen, Belgium

${ }^{10}$ Department of Cellular Therapy and Allogeneic Stem Cell Transplantation,

Karolinska University Hospital Huddinge, Stockholm, Sweden

${ }^{11}$ Department of Haematology, Amsterdam UMC Locatie VUmc, Amsterdam, NoordHolland, The Netherlands

${ }^{12}$ Department of Haematology, Leiden University Medical Center, Leiden, ZuidHolland, The Netherlands

${ }^{13}$ Department of Clinical Immunology and Rheumatology, University Hospital Centre Zagreb, Zagreb, Croatia

${ }^{14}$ Department of Internal Medicine, Rheumatology and Clinical Immunology,

University Hospital Wurzburg, Wurzburg, Bayern, Germany

${ }^{15}$ Department of Medicine, Haematology and Oncology, Ruhr University of Bochum, Faculty of Medicine, Bochum, Nordrhein-Westfalen, Germany

${ }^{16}$ Department of Rheumatology, University Hospital Ghent, Gent, Oost-Vlaanderen, Belgium 
${ }^{17}$ Unit for Molecular Immunology and Inflammation, VIB Inflammation Research Center (IRC), Gent, Oost-Vlaanderen, Belgium

${ }^{18}$ Department of Rheumatology, Medical Center-University of Freiburg, Freiburg,

Baden-Württemberg, Germany

${ }^{19}$ Rheumatology, Radboudumc, Nijmegen, Gelderland, The Netherlands

${ }^{20}$ Department of Rheumatology, Amsterdam UMC Locatie VUmc, Amsterdam, NoordHolland, The Netherlands

${ }^{21}$ Rheumatology, Leiden University Medical Center, Leiden, Zuid-Holland, The

Netherlands

${ }^{22}$ Department of Rheumatology, University Hospital Basel, Basel, Switzerland

Acknowledgements We would like to thank the Dutch and Belgian patient advisers for providing input in the study design and patient information. We acknowledge ZonMW, Boehringer Ingelheim, Miltenyi and the private funding partner for financially supporting this study.

Contributors JS is the coordinating investigator, had overall responsibility for the trial design and drafted the trial protocol and manuscript. JMvL is the principal investigator, had overall responsibility for the trial design and trial protocol, and contributed to the manuscript. AvdL, AvR, AV, DD, DW, EDL, EM, JH, JdV-B, KG, MM, MS, MV, NDP, PL, RH, RS, RV, TK, UW and VS contributed to trial design and the manuscript. PW is responsible for statistical and economic analysis. AM is the trial manager, JdV-B coordinates the substudies in this trial. Centre leads are AEV (Amsterdam), DW (Lund), EDL (Leuven), JH (Tubingen), JdV-B (Leiden), KG (Stockholm), MM (Zagreb), MS (Wurzburg), MV (Nijmegen), NDP (Milan), RS (Bochum), RV (Freiburg), UW (Basel) and VS (Ghent). All authors inputted to the trial protocol and commented on the manuscript.

Funding This work was supported by zonMW (grant number 848018 003) and an unrestricted grant from Boehringer Ingelheim (grant number S20011), Miltenyi Biotec and a private funding partner. VS is a Senior Clinical Investigator of the Research Foundation-Flanders (Belgium) (FWO) (1.8.029.15N). The FW0 was not involved in study design; collection, analysis and interpretation of data; writing of the report; and the decision to submit the manuscript for publication.

Competing interests None declared.

Patient consent for publication Not required.

Provenance and peer review Not commissioned; externally peer reviewed.

Supplemental material This content has been supplied by the author(s). It has not been vetted by BMJ Publishing Group Limited (BMJ) and may not have been peer-reviewed. Any opinions or recommendations discussed are solely those of the author(s) and are not endorsed by BMJ. BMJ disclaims all liability and responsibility arising from any reliance placed on the content. Where the content includes any translated material, BMJ does not warrant the accuracy and reliability of the translations (including but not limited to local regulations, clinical guidelines, terminology, drug names and drug dosages), and is not responsible for any error and/or omissions arising from translation and adaptation or otherwise.

Open access This is an open access article distributed in accordance with the Creative Commons Attribution Non Commercial (CC BY-NC 4.0) license, which permits others to distribute, remix, adapt, build upon this work non-commercially, and license their derivative works on different terms, provided the original work is properly cited, appropriate credit is given, any changes made indicated, and the use is non-commercial. See: http://creativecommons.org/licenses/by-nc/4.0/.

ORCID iD

Julia Spierings http://orcid.org/0000-0002-2546-312X

\section{REFERENCES}

1 Denton CP, Khanna D. Systemic sclerosis. Lancet 2017;390:1685-99.

2 Elhai M, Meune C, Boubaya M, et al. Mapping and predicting mortality from systemic sclerosis. Ann Rheum Dis 2017;76:1897-905.

3 Tyndall AJ, Bannert B, Vonk M, et al. Causes and risk factors for death in systemic sclerosis: a study from the EULAR scleroderma trials and research (EUSTAR) database. Ann Rheum Dis 2010;69:1809-15.

4 loannidis JPA, Vlachoyiannopoulos PG, Haidich A-B, et al. Mortality in systemic sclerosis: an international meta-analysis of individual patient data. Am J Med 2005;118:2-10.

5 Altman RD, Medsger TA, Bloch DA, et al. Predictors of survival in systemic sclerosis (scleroderma). Arthritis \& Rheumatism 1991;34:403-13.

6 Bulpitt KJ, Clements PJ, Lachenbruch PA, et al. Early undifferentiated connective tissue disease: III. outcome and prognostic indicators in early scleroderma (systemic sclerosis). Ann Intern Med 1993;118:602-9.

7 Burt RK, Milanetti F. Hematopoietic stem cell transplantation for systemic sclerosis: history and current status. Curr Opin Rheumatol 2011:23:519-29.

8 van Laar JM, Farge D, Sont JK, et al. Autologous hematopoietic stem cell transplantation vs intravenous pulse cyclophosphamide in diffuse cutaneous systemic sclerosis: a randomized clinical trial. JAMA 2014;311:2490-8.

9 Assassi S, Wang X, Chen G, et al. Myeloablation followed by autologous stem cell transplantation normalises systemic sclerosis molecular signatures. Ann Rheum Dis 2019;78:1371-8.

10 Eyraud A, Scouppe L, Barnetche T, et al. Efficacy and safety of autologous haematopoietic stem cell transplantation in systemic sclerosis: a systematic review of the literature. $\mathrm{Br} J$ Dermatol 2018;178:650-8.

11 Shouval R, Furie N, Raanani P, et al. Autologous hematopoietic stem cell transplantation for systemic sclerosis: a systematic review and meta-analysis. Biol Blood Marrow Transplant 2018;24:937-44.

12 Host L, Nikpour M, Calderone A, et al. Autologous stem cell transplantation in systemic sclerosis: a systematic review. Clin Exp Rheumatol 2017;35 Suppl 106:S198-207.

13 Smith V, Scirè CA, Talarico R, et al. Systemic sclerosis: state of the art on clinical practice guidelines. RMD Open 2019;4:e000782.

14 Kowal-Bielecka O, Fransen J, Avouac J, et al. Update of EULAR recommendations for the treatment of systemic sclerosis. Ann Rheum Dis 2017;76:1327-39.

15 van Bijnen S, de Vries-Bouwstra J, van den Ende $\mathrm{CH}$, et al. Predictive factors for treatment-related mortality and major adverse events after autologous haematopoietic stem cell transplantation for systemic sclerosis: results of a long-term follow-up multicentre study. Ann Rheum Dis 2020;79:1084-9.

16 Farge D, Burt RK, Oliveira M-C, et al. Cardiopulmonary assessment of patients with systemic sclerosis for hematopoietic stem cell transplantation: recommendations from the European Society for blood and marrow transplantation autoimmune diseases Working Party and collaborating partners. Bone Marrow Transplant 2017;52:1495-503

17 Carreras E, Mohty M, Kroger M. Hematopoietic Stem Cell Transplantation and Cellular Therapies. In: The EBMT Handbook. Springer Nature Switzerland AG, 2019.

18 Distler O, Assassi S, Cottin V, et al. Predictors of progression in systemic sclerosis patients with interstitial lung disease. Eur Respir J 2020;55:1902026.

19 Andréasson K, Saxne T, Bergknut C, et al. Prevalence and incidence of systemic sclerosis in southern Sweden: population-based data with case ascertainment using the 1980 Ara criteria and the proposed ACR-EULAR classification criteria. Ann Rheum Dis 2014;73:1788-92.

20 Vonk MC, Broers B, Heijdra YF, et al. Systemic sclerosis and its pulmonary complications in the Netherlands: an epidemiological study. Ann Rheum Dis 2009;68:961-5.

21 Khanna D, Berrocal VJ, Giannini EH, et al. The American College of rheumatology provisional composite response index for clinical trials in early diffuse cutaneous systemic sclerosis. Arthritis Rheumatol 2016;68:299-311.

22 Guideline for economic evaluations in healthcare. Available: https:// english.zorginstituutnederland.nl/publications/reports/2016/06/16/ guideline-for-economic-evaluations-in-healthcare

23 Spierings J, van Rhijn- Brouwer FCC, de Bresser JM. Treatment decision-making in hematopoietic stem cell transplantation in patients with poor prognosis diffuse cutaneous systemic sclerosis. Rheumatology 2020;59:1226-32. 